\title{
BIM-BASED HOLONIC SYSTEM FOR REAL-TIME PATHFINDING IN BUILDING EMERGENCY SCENARIO
}

\author{
Berardo Naticchia, Leonardo Messi, and Alessandro Carbonari \\ Polytechnic University of Marche, Ancona, Italy
}

\begin{abstract}
Emergency management can benefit from advanced information and communication technology (ICT), since they can support officers in charge with rescue operation to deal with urgent decision within a really short deadline. Further enhancement can derive from the application of holonic systems, inherited from the manufacturing industry, to tackle unforeseen scenario. The aim of this research is to develop a BIM-integrated technology for the real-time pathfinding during emergencies. A virtual reality platform, exploiting building topological data from the digital model, can support the standard emergency approach if an unexpected event voids the network of predetermined paths. The developed system has been tested in a large mixed-used building assumed as case study.
\end{abstract}

\section{Introduction}

\section{Building Management Systems}

Building automation systems (BAS), also known as building management systems (BMS), denote a wide range of computerized building control systems, from special-purpose controllers, to standalone remote stations, to larger system including central computer stations. A BAS comprises several subsystems which are connected in various ways to form a complete system. The system must be designed and engineered around the building itself to serve the services systems for which it is intended. Consequently, although the component parts used may be identical, no two systems are the same, unless they are applied to identical buildings with identical services and identical uses (Wang S., 2009). BMS, with their typical hierarchical structure, are usually able to reach their goals in an efficient way and with no faults, whereas they fail to stick to pre-determined targets in the presence of disturbances. In fact, traditional systems cannot pursue the assigned task if any unforeseen events occur. Their rigid structure makes it very difficult to tackle unexpected scenarios. As low-level modules have to consult higher hierarchy levels in case of a disturbance, their reactivity becomes weak. Furthermore, global decision-making is often based on obsolete information (Valckenaers et al., 2016). Building services include HVAC systems, electrical systems, lighting systems, fire systems, security systems and lift systems. In industrial buildings they may also include the compressed air, steam and hot water systems used for the manufacturing process (Wang S., 2009).
In this paper, fire and security systems for the emergency management will be studied in depth in order to overcome the limits of the traditional BMS. Emergency management, since directly affects safety of people, represents a relevant issue in each phase of building lifecycle, from a foresighted design of buildings and infrastructures to the elaboration of emergency plans according to specific regulations. Emergency scenarios are even more relevant as case studies, if the high frequency of unexpected events affecting them is considered. The traditional approach to the emergency management is based on a deterministic forecast of main scenarios. The emergency plan resulting from them has a key role, although it does not consider the totality of possible scenarios. As a consequence, it is regardless of contextual, changing and unexpected events that may happen and seriously affect the effectiveness of emergency measures. The limits of such a knowledge-based approach are confirmed by several examples of complications in the emergency operations. To name one, during the emergency response to the September 11, 2001 attack on the World Trade Centre, commanders on the scene were unable to communicate to '911' Public Service Access Points (PSAP) that people should evacuate the building (Ulieru et al., 2006). As a result, PSAP operators complied with New York City's standard operating procedure for hirise fires and advised callers to stay in impacted buildings. The ' 911 ' system was inadequate for handling a major disaster and could not adapt to the emergency. The final death toll 2749 may have been substantially reduced if the PSAP's were adaptive in coping with the overload. Moreover, commanders trying to evacuate fire fighters from the north tower during the World Trade Centre disaster were seriously hampered by ineffective radio communications; the final death toll 343 of New York fire fighters may also have been substantially reduced if the system controlling the radio communications was also adaptive (Ulieru et al., 2006). To name another example, the Grenfell Tower fire produced a high number of victims not only for technical reasons, due to the employment of a not proper cladding system and to a lack of separated fire boxes into the building, but also to a mistake in the emergency evaluation (Lane, 2018). The "stay put" strategy, led by a tardive declaration of the situation as a major incident with the consequent delay of one hour in the evacuation process, has revealed as a fatal mistake in the rescue operations (Lane, 2018). Furthermore, a traditional emergency plan does not facilitate the 
operational use. In fact, it remains as a plain-text document which is difficult to consult during an excited situation due to an oncoming danger. In other words, a way-out plan hung on the wall cannot help incisively to find a viable exit route. The fire in the Rhode Island station club represents an example of how a not profound knowledge of the building in which people were located affected the evacuation process: a study has demonstrated that people did not use alternative ways-out since they ignored their presence (Grosshandler et al., 2005). While the main exit doors were obstructed by the smoke presence, there were no indication to use alternative paths to escape from the building; therefore, the evacuation process was affected by a fatal delay (Grosshandler et al., 2005).

Most traditional studies about pathfinding for fire emergency evacuation considers only 2D navigation and find it difficult to respond to the ever-changing emergency circumstances due to their limitations in utilizing real-time building information. Recent researches introduce the BIM-based pathfining to detect traditional exit routes; a common feature is the manual pre-processing phase which extracts from the IFC project all the needed building information. Amongst them, Wang et al. (2014) focus on the evacuation training; $\mathrm{Xu}$ et al. (2017) compares the results from a 2D and 3D spatial navigation; Ismail et al. (2017) applies graph databases and graph theory concepts for advance analysing of BIM models as well as pathfinding.

Starting from the above-mentioned shortcomings and previous researches, the current one proposes a contingent approach which exploits a BIM-based holonic technology to overcome the limits of the traditional BMS in real emergency scenarios. The building digital model, imported in an environment endowed with physics engine, provides all the necessary data for $3 \mathrm{D}$ ways-out detection in real-time. The developed holonic architecture introduces resilience and emergent collaboration in order to tackle unexpected events by means of alternative and unconventional escape solutions. This paper is organized as follows. The following section provides a description of the system architecture. Then, a section describes the Virtual Reality Platform. The next one provides a description of the BIM integration. Afterwards the Bayesian Selector is described. Another section provides a description of Real-Time Pathfinding and shows the simulations results. The last one is devoted to conclusion.

\section{Holonic Theory}

The holonic concept, which is the basis of holonic management systems, is the key enabler to tackle unexpected events and overcome the limits of the traditional BMS. The holonic theory was introduced in 1967 by Koestler (Koestler, 1967) to explain the evolution of biological and social systems. Likewise, in the real world, where almost everything is at the same time a part and a whole, each holon can be part of another holon (Valckenaers et al., 2016). In fact, the word holon is the combination of "holos", which in Greek means "whole", and the suffix "on", which suggests a part (Wang L. et al., 2015), (Verstraete et al., 2006), (Giret et al., 2004). In the manufacturing field, holons are autonomous and cooperative building blocks, since they can both control the executions of their own strategies and develop mutually acceptable plans (Valckenaers et al., 2016). Furthermore, holons consist of an information-processing part and often a physical-processing part (Valckenaers et al., 2016), (Wang L. et al., 2015), (Verstraete et al., 2006). The former is responsible for high-level decision making, collaborating and negotiating with humans and other holons, while the latter is a representative of its linked physical component and responsible for transferring decisions and instructions to it (Wang L. et al., 2015). According to Koestler, a holonic system or holarchy is then a hierarchy of self-regulating holons that function (i) as autonomous wholes in supra-ordination to their parts, (ii) as dependent parts in subordination to control at higher levels, and (iii) in coordination with their local environment (Valckenaers et al., 2016), (Koestler, 1967), (Giret et al., 2004). Therefore, holonic architecture combines high and predictable performance, which distinguishes hierarchical systems, with the robustness against disturbances and the agility typical of heterarchical systems (Verstraete et al., 2006). In this way, systems' resilience is guaranteed. Holonic management systems, which have been successfully applied in the manufacturing field, can constitute a novel technology to tackle unforeseen scenario variations. Indeed, the autonomy and cooperation of their elementary units, the holons, makes it possible to avoid the rigid structure of hierarchical systems and therefore respond quickly to disturbances (Valckenaers et al., 2016).

\section{System Architecture}

The architecture of the developed holonic system, depicted in Figure 1, supports fire emergency management and rescue operation, detecting the most effective way-out. Its aim is not to substitute the actual approach foreseen by regulations, rather to enhance the standard emergency plan, detecting unconventional path to exit the building, if an unexpected event occurred. The architectural principles are shortlisted as follows:

- Real-time effectiveness: it must regard both the information flow and the decision-making process. Since the system is continuously evolving, especially in complex scenarios like emergencies, it is not feasible to represent all the changing status it assumes, in order to provide the proper solution to the occurring situations. Moreover, the response of the system must be sufficiently reactive in order to result effective to face the situations that are in place.

- Proactive and unconventional problem solving: on the basis of the information gathered in a real-time manner, in order to be sufficiently resilient, the system must be capable to extract the information useful to reach its objectives also by general data, as well as to provide escape solutions with the employment of unconventional means. 
- Resilience: the system must be reactive and adaptive to the new possible configurations that may occur, without compromising its primary function of managing the emergency scenario. Failure, interruptions, damages to the standard communication backbone systems, as well as injuries or obstructions to the usual evacuation means, must not impede the main objective of keeping people safe during emergencies.

- Emergent cooperation: the system architecture embodies the capability of the holons to cooperate in temporary associations, namely the holarchies, introducing the "emergent cooperation". This concept can be described using a metaphor: the behaviour of these agents is similar to the one belonging to the birds of a flock; no one is able to manage flock's shape and dimension, but everyone takes care of maintaining flock's trajectory, flock's speed and minimum distance from its fellows. Although no one of the birds has a complete view of the scene, the behaviour described above is the result of a "emergent collaboration". This kind of cooperation is not so onerous for birds because it is supposed to be integrated within their DNA and, therefore, instinctive. The same functioning characterizes the agents inside the developed architecture.

The system architecture is composed by different layers interacting each other according to a publish/subscribe scheme, whose central concept is the notion of topic: each node/layer of the architecture subscribes and some other publishes information on the topic. In other words, the topic is simply the database content itself without intermediate language adapters: the unique language is the database language and its queries. Hence, the architecture layers behave like a human being that only subscribes to interesting events/information and publishes relevant events/information, which are specifically useful for the occurring situation, complying to the actual needs (Bonci et al., 2017).

The different layers can be clustered by function according to the PROSA reference architecture (Valckenaers et al., 2016), a conceptual architecture for manufacturing control, that has shown great potentialities to be applied also in different context. The acronym PROSA stands for product-resource-order-staff architecture and refers to the different types of holons. Three basic types of holons can be distinguished: product holons, resource holons, and order holons. Staff holons are optional and can be added to provide the other holons with expert knowledge (Valckenaers et al., 2016). In PROSA, the holons' physical part belongs to the world of interest, that is the part of reality which falls within a certain scope relevant for the application (Verstraete et al., 2006). In Figure 1, the PROSA analogy is highlighted identifying resource, product and order holons. Finally, the environment is the real-world scenario where the holons operate, namely the fire occurring and the countermeasures to be taken at the end of the elaboration process. The highest architecture layers, namely the General Safety Objectives and the Generic Metaplans, correspond to the order holon, which

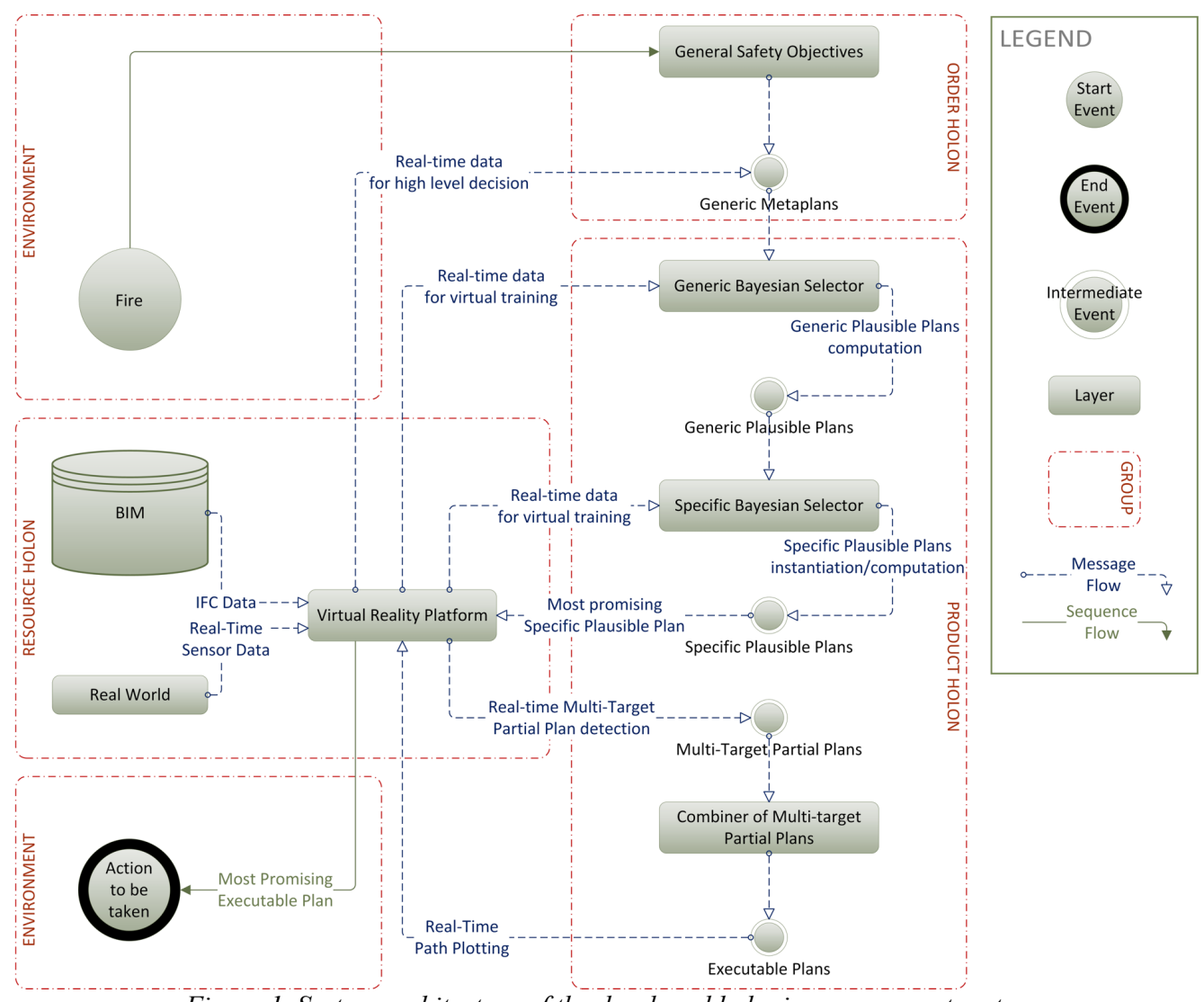

Figure 1. System architecture of the developed holonic management system. 
in the manufacturing system represents a task that needs to be executed, for example the delivery of a package (Valckenaers et al., 2016). The General Safety Objectives represent the always valid target: "save all the people inside the building". As a consequence, the Generic Metaplans are triggered and fed with real-time data from the Virtual Reality Platform. The output of this elaboration are the general processes to be executed, which may be represented by the generic instruction "stay in/go out" and are published to the following layer. Thanks to the generality of the high-level setting, the resilience architectural principle is guaranteed and the resulting system is applicable for every building, without the need to configure it manually every time. This is a key feature that distinguishes the developed architecture from classic BMS.

\section{Virtual Reality Platform}

The Virtual Reality Platform (VRP) is the heart of the resource holon which corresponds, in the logistic context, to resources like all transport means and material handling equipment (Valckenaers et al., 2016). In the developed system the VRP is implemented using the Unity 3D game engine. It has been selected as the most suitable tool because of the following characteristics:

- high interoperability with other software, including the capability to integrate several functional mockups afferent to different engineering disciplines;

- $\quad$ presence of a physics engine that provides physical behaviour to the components of the scene, basically the correct acceleration and the affections by collisions, gravity and other forces, making the simulation of a great likelihood with the real world;

- possibility to introduce artificial intelligence by scripts, based on C\# or Java programming languages, or by means of visual programming.

In other words, Unity 3D offers an extremely realistic environment to simulate a fire emergency scenario of the case study and building occupants' behaviour using artificial intelligence. The VRP constitutes a dynamic hub able to collect IFC data from BIM and real-time data from pervasive sensors distributed in the real world. The interconnection with the BIM software Autodesk Revit has been established through an IFC Loader, based on the IFCEngine DLL Library (RDF Ltd., 2006). This component makes it possible to easily import topological information, materials properties and all semantic information from the digital model, once exported in IFC format. The interconnection with sensors, which has not been tested in this research step, is one of future developments. The technological solution to this issue is ASP.NET Core SignalR, an open-source library that simplifies adding real-time web functionality to apps. Real-time web functionality enables server-side code to push content to clients instantly (Microsoft Corp., 2018). As depicted in Figure 1, the VRP acts as a collector of heterogeneous data and represents the proper environment where the required knowledge can be produced and published. In this way, real-time data are available for each subscribing layer of the architecture.

A* Pathfinding Project Pro (A*PPP) (Granberg, 2017), is one of the most popular dedicated pathfinding Unity assets and the benchmark for quality pathfinding in Unity. Unlike Unity default NavMesh (Unity 3D Doc., 2018), it supports dynamic updates of the graphs at runtime. Hence, A*PPP, applying the $\mathrm{A}^{*}$ algorithm, detects in real-time the most effective way-out. If the usual escape route is obstructed by smoke, fire, collapsed building elements or other kinds of accidents, the $\mathrm{A}^{*}$ algorithm can detect unconventional ways-out through internal doors.

\section{BIM integration}

The system architecture integrates the BIM technology with the aim to improve the context awareness during the emergency management. The building digital model, coherently updated during the whole lifecycle, provides in real-time accurate building information, that goes further beyond the static and poor information usually contained in emergency plans. In fact, a BIM organizes and stores contextual, geometrical and materials information, facilitating the consultation at any time, especially by users and rescuers during excited moments. Building digital information lay the basis for automatic detection of alternative path outwards, whether pre-determined standard routes result unviable. Moreover, BIM is proposed not only as a comprehensive provider of the building data, but it is subjected to a proper semantic enhancement: some building elements can be exploited in an unusual way in respect of their main purpose and become evacuation means in an unconventional manner. These contributions appear even more relevant, if we consider the possibility to have random visitors inside building affected by a poor awareness of the spatial distribution. Furthermore, the building information model, once imported in the VRP, can be updated with data about the monitored spaces deriving from sensors deployed on site. Real-time location systems (RTLS), integrated with BIM, provides real-time information about a relevant aspect like the position of people trapped during an emergency. As mentioned in the previous section, BIM data can be leveraged within the VRP by means of an IFC Loader, completing the role of the resource holon.

\section{Bayesian Selector}

The Bayesian Selector (BS) is conceived as the translation into a Bayesian network of an expert's knowledge. Bayesian networks, which constitute a powerful mean to represent phenomena affected by a high level of uncertainty, are a probabilistic graphical model that uses Bayesian inference for probability computations. They aim to model conditional dependence, and therefore causation, by representing conditional dependence by edges in a directed graph. Through these relationships, one can efficiently conduct inference on the random variables in the graph through the use of factors (Soni, 2018). 


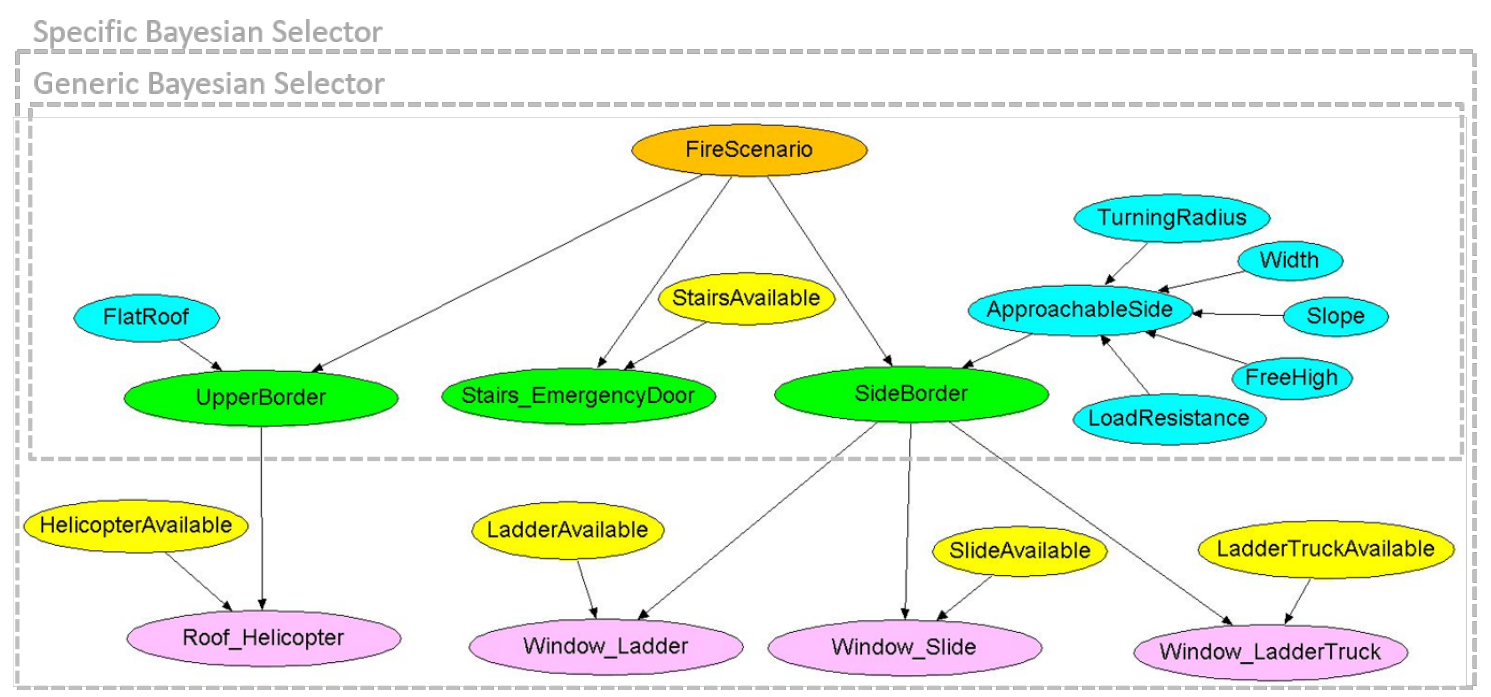

Figure 2. Bayesian Selector.

In the fire emergency management, the BS, fed by realtime data from the VRP, detects which strategy or plan is probabilistically the most effective to exit safely the building in fire. As shown in Figure 1, it is composed by a generic part and a specific one, respectively linked to Plausible Plans and Multi-Target Partial Plans. The Generic BS suggests the most effective preliminary escape strategy (called also Generic Plausible Plan) amongst those ones valid for any building. The Specific BS detects the most promising escape plan (called also Specific Plausible Plan), instantiated for the analysed building. All these layers, according to the PROSA analogy, constitute the product holon, which contains the knowledge on how instances of a specific task type (represented by order holons) can be executed by the resources (Valckenaers et al., 2016). In Figure 2, BS's orange node, namely "Fire Scenario", constitutes a Boolean variable, which notices the occurring fire. Green nodes represent the Generic Plausible Plans; for example, "Stairs and Emergency Door" represents all the exit routes which exploit stairs and emergency doors.

They comprise not only the standard exit route suggested by the emergency plan, but also alternative and unconventional ways-out through internal doors connecting adjacent rooms. Purple nodes are the Specific Plausible Plans, automatically instantiated for the case study building exploiting the technology described in (Bonci et al., INDIN 2018) and (Bonci et al., ETFA 2018) and on the base of real-time data published by the VRP (for example, "Roof and Helicopter" stands for "lead people to the roof and pick up them by a helicopter"). Finally, blue nodes represent building's features (for example "Flat Roof" stands for "is the building's roof flat?") whereas yellow ones represent resource availability (for example "Stairs Available" stands for "are the building's stairs available/viable?").

\section{Real-Time Pathfinding}

In order to implement and test the developed architecture, the mixed-use building Eustachio, located in Ancona

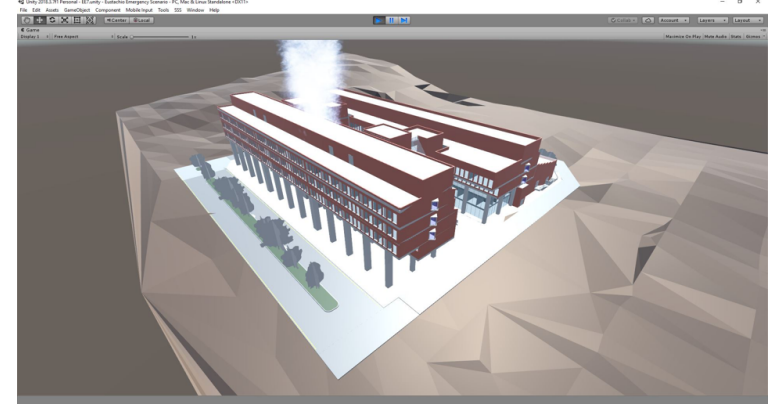

Figure 3. BIM of Eustachio loaded in Unity $3 D(V R P)$ in order to simulate a fire scenario.

(Italy), has been chosen. The building belongs to a major complex occupied by the Faculty of Medicine of Polytechnic University of Marche. This eight storeysbuilding presents a quite regular shape with two major blocks on the north and south side, containing spaces devoted to heterogeneous scopes: classrooms, scientific laboratories, administrative offices for students, a library, books storage rooms, services. The two main blocks are connected by the two double stairwells of the building. The choice has fallen on this edifice for its characteristic of public building with several uses that may involve variable flow of different people inside it: estimated between 100 and 2320 individuals and characterized by several age ranges and, more specifically, owning a different level of knowledge of the building. Finally, the presence of inflammable substances in several laboratories leads to simulate the fire scenario in this building (see Figure 3), since this is one of the most common and disruptive emergency situations, often affected by unexpected events and mistakes in the management of the evacuation process.

The Eustachio's standard emergency plan, provides a network of pre-determined ways-out, indicated on site by dedicated panels. For example, as shown in Figure 4, the black line points out the route to reach the stairwell and so the safe place outside. If a fire occurs in a room and expands up to the hallway, the standard exit route is blocked and the user cannot rely on the emergency plan to 
reach the closest safe place (see Figure 4). In cases like this, it is not easy for the user to search for an alternative way-out, because he could be a random visitor with limited knowledge of the building. Moreover, the smoke, which expands throughout the hallway, limits visibility and the possibility to get oriented, also in a familiar place.

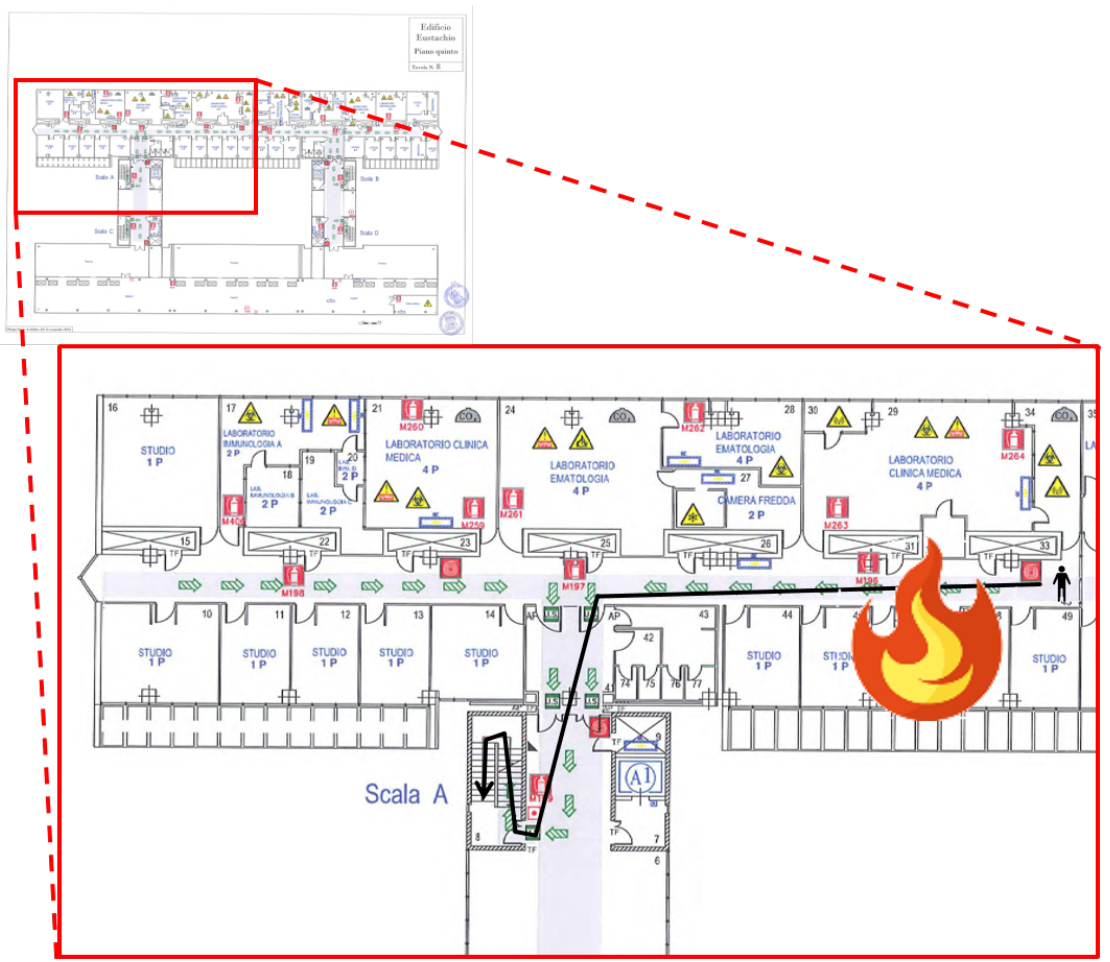

Figure 4. Eustachio's emergency plan: example of standard way-out (black line) obstructed by fire and smoke.

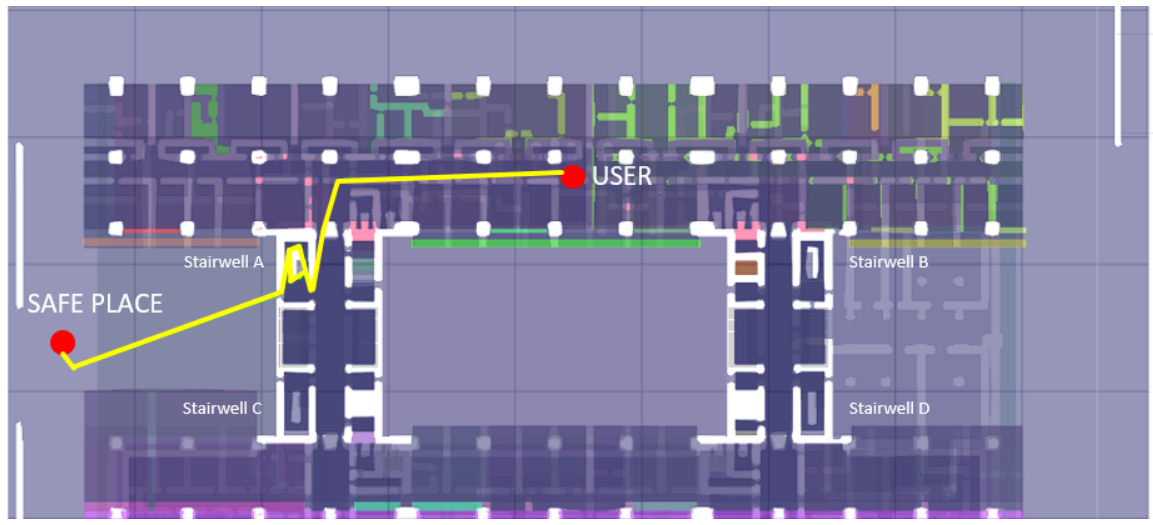

Figure 5. Standard way-out detected by $A * P P P$, coherently to the emergency plan for a normal scenario.

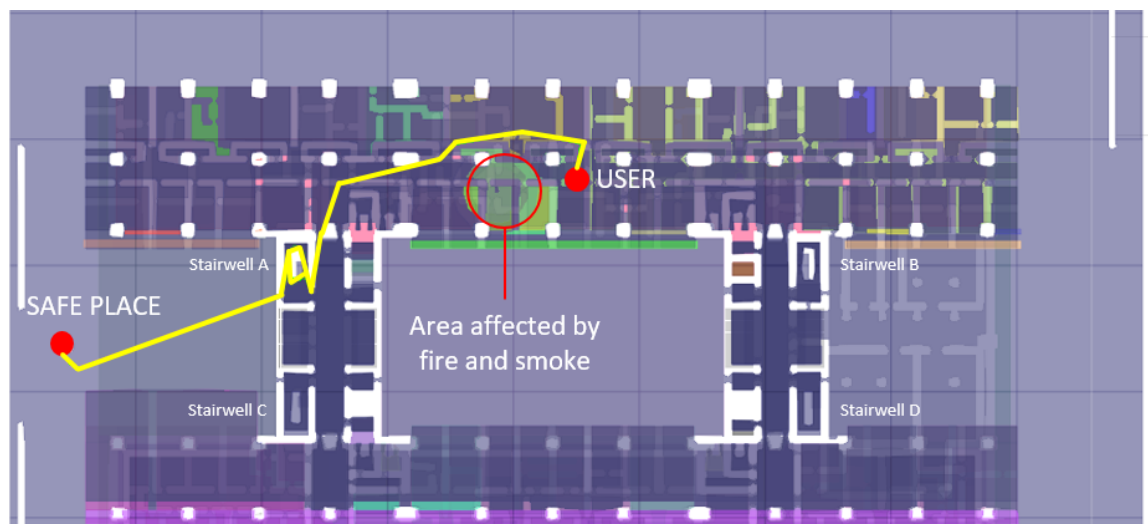

Figure 6. Alternative way-out detected by $A$ *PPP for an unforeseen fire scenario. 
The developed system, utilizing building updated data, hosted in BIM and loaded into the VRP (see Figure 3), is able to carry out a real-time pathfinding to tackle emergency scenarios, unforeseen by the standard emergency plan. The switch from the standard way-out (see Figure 5) to the alternative one (see Figure 6) occurs automatically, as soon as the fire is detected by sensors and simulated in the VRP. A*PPP, detecting all the connected floors surfaces (highlighted by purple color in Figure 5 and Figure 6), finds rooms internally connected by doors. The alternative path circumvents the zone affected by fire and smoke and leads the endangered users towards the closest stairwell. In order to readily inform them about the everchanging plan, a dynamic signage system (Galea et al., 2014) and a directional sound system (Plocher et al., 2011) can be integrated. If signage disappears behind the smoke, directional sound system continues to be effective. In fact, it uses verbal messages and a broadband multifrequencybased noise that allows the brain to work out exactly where the source of this sound is located. At the same time, the fire commander, equipped by a ruggedized tablet (Luyten et al., 2006), is informed about the evolving scenario and able to coordinate other firefighters by radio communication.

\section{Conclusions}

The holonic management system, described in this paper, improves the usual emergency management approach, leveraging more updated and significant information. Since a complete representation of every incidental events that may occur is not feasible, the current work proposes to move from a knowledge-based approach to a contingent one. Exploiting data from BIM and deployed sensors, the VRP computes real-time pathfinding to tackle scenarios unforeseen by the standard emergency plan. The approach described in the current paper is able to detect in real-time alternative and unconventional ways-out to be applied during emergency. Applying holonic theory and Bayesian inference, an effective emergent collaboration between low level holons makes it possible to get away safely; it can be done if a semantic enrichment labels some building elements as evacuation means. The developed system architecture tested in a large mixed-use public building, shows its potentiality through the contribution given to officers in charge of emergency management. More in details, the results of real-time pathfinding, support endangered users as well as rescuers, dealing with urgent decision within a really short deadline.

In future researches, a continuous update of IFC data will be tested in order to guarantee system functionality also after building refurbishment. Moreover, the connection between sensors and VRP and the implementation of the BS represent relevant matters of work in order to automate the functioning of the whole system.

\section{Nomenclature}

ICT (Information and Communication Technology),

BIM (Building Information Modeling),
BAS (Building Automation Systems), B

MS (Building Management Systems, HVAC (Heating, Ventilation and Air Conditioning), PSAP (Public Service Access Points), VRP (Virtual Reality Platform), BS (Bayesian Selector), PROSA (Product Resource Order Staff Architecture), IFC (Industry Foundation Class), A*PPP (A* Pathfinding Project Pro).

\section{Acknowledgments}

This research work was supported by the EU funded project "ENergy aware BIM Cloud Platform in a COsteffective Building REnovation Context' - ENCORE" (H2020, Grant Agreement no. 820434).

\section{References}

Bonci A., Pirani M., Longhi S. (2017). A Database-Centric Framework for the Modeling, Simulation and Control of Cyber-Physical Systems in the Factory of the Future, Journal of Intelligent Systems, 27, 10.1515/jisys-2016-0281.

Bonci A., Pirani M., Cucchiarelli A., Carbonari A., Naticchia B., Longhi, S. (2018). A Review of Recursive Holarchies for Viable Systems in CPSs, in: Proceedings of the 2018 IEEE 16th International Conference on Industrial Informatics INDIN, 37-42, 10.1109/INDIN.2018.8472055.

Bonci A., Pirani M., Carbonari A., Naticchia B., Cucchiarelli A., Longhi S. (2018). Holonic Overlays in Cyber-Physical System of Systems, in: Proceedings of the 2018 IEEE 23rd International Conference on Emerging Technologies and Factory Automation ETFA, 1240-1243, 10.1109/ETFA.2018.8502586.

Galea E.R., Xie H. and Lawrence P.J. (2014). Experimental and Survey Studies on the Effectiveness of Dynamic Signage Systems, Fire Safety Science 11: 1129-1143, 10.3801/IAFSS.FSS.11-1129.

Giret A., Botti V. (2004). Holons and Agents, Journal of Intelligent Manufacturing, 10.1023/B:JIMS.0000037714.56201.a3.

Granberg A., A* Pathfinding Project (2017). Available online (last accessed 9.1.2019): https://arongranberg.com/astar/\#.

Grosshandler W.L., Bryner N., Madrzykowski D., Kuntz. K. (2005). Report of the Technical Investigation of The Station Nightclub Fire, National Institute of Standards and Technology.

Ismail A., Nahar A. (2017). Application of graph databases and graph theory concepts for advanced analysing of BIM models based on IFC standard, in: Proceedings 24th International Workshop on 
Intelligent Computing in Engineering, Nottingham, ISBN: 9781510843455.

Koestler A. (1967). The Ghost in the Machine, The Macmillan Company, Hutchinson, ISBN: 0-14019192-5.

Lane B., New Civil Engineer (2018). Available online (last accessed 10.12.2018): https://www.newcivilengineer.com/latest/grenfellexpert-report-cites-catastrophicflaws/10031720.article.

Luyten K., Winters F., Coninx K., Naudts, D., Moerman I. (2006). A Situation-Aware Mobile System to Support Fire Brigades in Emergency Situations, OTM Workshops, 10.1007/11915072 105.

Microsoft Corp., Introduction to ASP.NET Core SignalR (25.04.2018), available online (last accessed 15.3.2019): $\quad$ https://docs.microsoft.com/itit/aspnet/core/signalr/introduction?view=aspnetcore2.2 .

T. Plocher, Z. J. Jin, F.-Y. D. Chan (2011). Using Sound Patterns to Enhance Directional Sound for Emergency Route Guidance, in: Proceedings of the 14th International Conference on Human-Computer Interaction, Orlando, 10.1007/978-3-642-21616-9_37.

RDF Ltd., IFCEngine DLL Library (2006). Available online (last accessed 9.1.2019): http://www.ifcbrowser.com.

Soni D., Introduction to Bayesian Networks (2018). Available online (last accessed 8.1.2019): https://towardsdatascience.com/introduction-tobayesian-networks-81031 eeed94e.

Valckenaers P., Van Brussel H. (2016). Design for the Unexpected, From Holonic Manufacturing Systems towards a Humane Machatronics Society, Butterworth-Heinemann, ISBN: 978-0-12-803662-4.

Ulieru M., Worthington P. (2006). Autonomic risk management for critical infrastructure protection. Integrated Computer-Aided Engineering, 13, 63-80, 10.3233/ICA-2006-13105.

Unity 3D Doc. (2018). NavMesh, available online (last accessed 15.1.2019): https://docs.unity3d.com/ScriptReference/AI.NavMes h.html.

Verstraete P., Saint Germain B., Hadeli K., Valckenaers P., Van Brussel H. (2006). On applying the PROSA reference architecture in multi-agent manufacturing control applications, Multiagent Systems and Software Architecture, in: Proceedings of the Special Track at Net.ObjectDays, Erfurt.
B. Wang, H. Li, Y. Rezgui, A. Bradley, H. N. Ong (2014). BIM Based Virtual Environment for Fire Emergency Evacuation, The Scientific World Journal, $10.1155 / 2014 / 589016$.

Wang L., Haghighi A. (2015). Combined strength of holons, agents and function blocks in cyber-physical systems, Journal of Manufacturing Systems, 10.1016/j.jmsy.2016.05.002.

Wang S. (2009). Intelligent buildings and building automation. Routledge, ISBN: 0-203-89081-7.

Xu M., Wei S., Zlatanova S., Zhang R. (2017). BIM-based indoor path planning considering obstacles, ISPRS Annals of the Photogrammetry, in: Remote Sensing and Spatial Information Sciences, Vol. IV-2/W4, 417423, 10.5194/isprs-annals-IV-2-W4-417-2017. 